Background and aims B-type natriuretic peptide (BNP) and Nterminal-pro-BNP (NTproBNP) have been shown to correlate with the size of patent ductus arteriosus (PDA) in preterm infants. We investigated whether BNP or NTproBNP is more accurate for assessment of a PDA.

Methods Prospective observational study. Preterm infants born. Results 60 infants were recruited, 58 had complete datasets. The cohort's mean (SD) gestational age was $27^{3}\left(2^{2}\right)$ weeks and had a mean (SD) birth weight of 1032 (315) grams. 46 (79.3\%) infants had a PDA with a mean (SD) PDA diameter of 3.2 (0.9) mm. Median (IQR) BNP levels: 486.5 (219-1316) pg/ $\mathrm{ml}$ for infants with PDA, 190 (95.5-514.5) $\mathrm{pg} / \mathrm{ml}$ for infants without PDA. Median (IQR) NTproBNP levels: 10858.5 (6319-42108) pg/ml for infants with PDA, and 7488 (336314227.5) $\mathrm{pg} / \mathrm{ml}$ for infants without PDA. Both BNP and NTproBNP showed a significant correlation with PDA size in this cohort: $\mathrm{BNP} \mathrm{R}=0.35$ ( $\mathrm{p}=0.0066)$; NTproBNP $\mathrm{R}=0.31$ $(\mathrm{p}=0.018)$.

Conclusion BNP and NTproBNP were closely correlated to PDA size. Both markers were useful for assessment of PDA size in this cohort of very preterm infants.

\section{0-032 SEROTONIN IS A SELECTIVE VASOCONSTRICTOR OF CHICKEN EMBRYO DUCTUS ARTERIOSUS}

L van Zogchel, E Villamor. Pediatrics, Maastricht University Medical Center, Maastricht, Netherlands

\subsection{6/archdischild-2014-307384.101}

Introduction Decreased platelet number and/or function have been related to patent ductus arterious (DA). Activated platelets release vasoactive products, including serotonin $(5-\mathrm{HT})$ that might be relevant for DA homeostasis DA. The chicken embryo has emerged as a suitable model for the study of DA vascular biology. In the present study, we investigated the possible vasoactive role of 5-HT in the chicken DA.

Methods Rings of the DA of 15- to 20-d-old chicken embryos (total incubation time 21-d) were studied in a wire myograph. The response to 5-HT was investigated under different $\mathrm{O}_{2}$ tensions $(3,7$, and $74 \mathrm{kPa})$. The responses to the $5-\mathrm{HT}_{1 \mathrm{~B} / \mathrm{D}}$ receptor

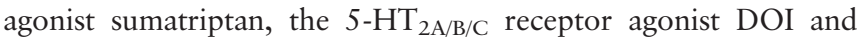
the selective serotonin reuptake inhibitors fluoxetine and sertraline were also investigated.

Results 5-HT (10 nM-0.3 mM) contracted the pulmonary side of the DA (PulmDA) in a concentration-dependent manner. By contrast, 5-HT induced negligible contractions in the vessels that surround the PulmDA (i.e., the pre- and post-ductal pulmonary arteries, and the aortic side of the DA). 5-HT-induced contraction increased with development (15-d >17-d >19$\mathrm{d}=20-\mathrm{d}) \cdot \mathrm{O}_{2}$ tension did not affect 5-HT-induced contraction but elimination of extracellular calcium completely abolished it. Sumatriptan and DOI also contracted the PulmDA in a concentration-dependent manner. By contrast, fluoxetine and sertraline evoked contractions at very high concentrations $(>0.1 \mathrm{mM})$.

Conclusions Our data indicate that 5-HT receptors are functionally present in the chicken DA and suggest that plateletderived 5-HT may play a pivotal role in the postnatal closure of the DA.

\begin{tabular}{lclll} 
Abstract 0-033 Table 1 & \multicolumn{5}{c}{ Clinical outcomes } \\
\hline & \multicolumn{5}{c}{ Rate (\%) } & p-value* & OR (Cl95\%) \\
\hline Survival-without-morbidity & P2 & 53.4 & NS & $1.34(0.70-2.57) \dagger$ \\
& P1 & 46 & & \\
Mortality & P2 & 18.2 & NS & $1.05(0.45-2.45)$ \\
& P1 & 17.5 & & \\
Chronic lung disease & P2 & 12.5 & $<0.05$ & $0.39(0.15-0.98)$ \\
Retinopathy ( $\geq 3)$ & P1 & 26.9 & & \\
& P2 & 12 & NS & $1.31(0.41-4.15)$ \\
Necrotizing enterocolitis & P1 & 9.4 & & \\
& P2 & 5.1 & $<0.05$ & $0.28(0.08-0.96)$ \\
Intraventricular haemorrhage $(\geq 3)$ & P1 & 16.1 & & \\
& P2 & 25 & NS & $0.90(0.43-1.88)$
\end{tabular}

P1 aggressive, P2 conservative

*Chi-square

tAdjusted for gestational age, sepsis and days on mechanical ventilation (logistic regression)

\section{0-033 CONSERVATIVE APPROACH TO PATENT DUCTUS ARTERIOSUS IN VERY LOW BIRTH WEIGHT INFANTS}

${ }^{1} \mathrm{C}$ Borras-Novell, ${ }^{2} \mathrm{~V}$ Aldecoa, ${ }^{2} \mathrm{M}$ Domingo, ${ }^{2} \mathrm{C}$ Figaró, ${ }^{1} \mathrm{~J}$ Moreno, ${ }^{1} \mathrm{~A}$ Riverola. ${ }^{1}$ Neonatology, Hospital Sant Joan de Déu, Barcelona, Spain; ${ }^{2}$ Neonatology, Parc Taulí Sabadell, Sabadell, Spain

\subsection{6/archdischild-2014-307384.102}

Background and aims Management of patent ductus arteriosus (PDA) is still a dilemma. We aimed to prove that a more conservative approach of PDA is equally effective without increasing morbidity-mortality in preterm infants.

Methods From January 2009 to December 2013 clinical charts of preterm $<31$ weeks admitted into two NICUs with clinical/ echocardiographic PDA were analysed. In January 2011 management was changed. In the first period (P1), patients who failed medical treatment underwent surgical ligation; in the second (P2), only those with cardiopulmonary compromise (mainly those that could not be weaned from ventilator). We compared survival-without-morbidity, defined as patients discharged without chronic lung disease, severe retinopathy, necrotizing enterocolitis or severe intraventricular haemorrhage.

Results Patients in P1 $(n=63)$ and P2 $(n=88)$ had similar clinical characteristics. Significant lower rates of medical $(85.7 \%$ vs $56.8 \%)$ and surgical treatment $(33.9 \%$ vs $14.3 \%)$ were observed in P2. No differences in survival-without-morbidity were observed (Table 1). In P2, 19.4\% patients showed PDA at discharge.

Conclusions A conservative approach in preterm with PDA can avoid medical/surgical treatment and its side effects, without changes in survival-without-morbidity.

\section{0-034 HALF SYSTOLIC DECAY TIME (1/2SDT) OF DUCTAL FLOW MEASURED BY ECHOCARDIOGRAPHY WOULD PREDICT NEED FOR TREATMENT OF PATENT DUCTUS ARTERIOSUS (PDA) IN EXTREMELY PREMATURE NEONATE}

${ }^{1}$ A Kulkarni, ${ }^{2} \mathrm{JS}$ Carvalho, ${ }^{1} \mathrm{~J}$ Richards. ${ }^{1}$ Neonatology, St George's Hospital NHS Trust, London, UK; ${ }^{2}$ Fetal Medicine, St George's Hospital NHS Trust Royal Brompton Hospital NHS Trust, London, UK

10.1136/archdischild-2014-307384.103 